\title{
Country Report: The Report of Transplant Activity and Outcome During the First 12 Months at the First National Hematopoietic Stem Cell Transplant Center in Sri Lanka
}

P Abeysinghe1, L Morawaka², S Gunasekara ${ }^{1}$, S Suresh ${ }^{1}$, PWDCC Jayathilake ${ }^{1}$, PHSP Udara ${ }^{1}$, PPR Siriwardena ${ }^{1}$, S Milliken ${ }^{3}$, J Moore ${ }^{3}$, DDF Ma ${ }^{3}$

${ }^{1}$ National Cancer Institute, Sri Lanka, ${ }^{2}$ National Blood Transfusion Service, Sri Lanka, ${ }^{3}$ Department of Haematology and BM Transplantation, St. Vincent's Hospital Sydney, NSW, Australia

Abstract

Sri Lanka is a developing country with a population of 21 million. Nation Cancer Institute ( $\mathrm{NCl)}$ is the largest tertiary cancer centre with universal health coverage. Absence of hematopoietic stem cell transplant (HSCT) was a major deficiency as most patients cannot afford transplant abroad. In 2013-14, NCl decided to establish the first National HSCT center in collaboration with St. Vincent's Hospital Sydney. Eventually, the first Autologous HSCT was established at $\mathrm{NCl}$ in December 2016.

Patients with multiple myeloma (MM) in very good partial remission or better remission, and patients with relapsed Non-Hodgkin's lymphoma (NHL) and Hodgkin lymphoma (HL), who were less than 65 years with good performance status were selected. Stem cells were mobilized with cyclophosphamide and G-CSF and the products were cryopreserved. Melphalan was the conditioning regime for MM while BEAM was used for HL and NHL.

Twenty autologous transplants were performed in the first year. Mean age was 47 years (range: 17-62) and male to female ratio was 3: 2 . There were $17 \mathrm{MM}$ and one each of $\mathrm{NHL}, \mathrm{HL}$ and POEMS syndrome patients. Median CD34+ stem cells collected was $12.72 \times 10^{6} / \mathrm{kg}$ (range: $3-31$ ) and median infused cell dose was $4.07 \times 10^{6} /$ kg (range: 2-7.4).

Median engraftment day was 13 (range: 11-19) and median hospitalization was 16 days (range: 14-20). All developed febrile neutropenia and Grade II thrombocytopenia. Zero transplant-related mortality was observed with acceptable morbidity. At the median follow-up of 47 weeks, the overall survival was $100 \%$ with all the patients still in remission.

Key words: Multiple myeloma, Autologous stem cell transplant, Sri Lanka

Submitted May 29, 2019; Accepted August 25, 2019; Published online November 25, 2019, Issued online November 25, 2019

Correspondence: Prasad Abeysinghe, National Cancer Institute, Cancer Hospital Road, Maharagama 10280, Sri Lanka,

E-mail: prasadabeysinghe@hotmail.com

\section{Introduction}

Sri Lanka, an island in the Indian Ocean with an area of $62,705 \mathrm{~km}^{2}$ has a population of 21 million. It is a developing country in Southeast Asia. It is divided into 9 provinces, 25 districts and 324 divisional secretariats (Figure 1). In Sri Lanka, the public sector provides free universal health coverage. The Department of Health and the provincial health sector covers the whole range of curative and preventive services. Life expectancy at birth is 72 years for males and 78 years for females (2016).
Total expenditure on health per capita is $\$ 369$ (3.5\% of GDP). Doctor-population ratio is 1.04 per 1,000 population $(70 \% \text { are employed in the public sector })^{1}$.

National Cancer Institute (NCI) is the only cancer hospital of Sri Lanka, established in 1958, under the government health services. The institution is situated in greater Colombo area and provides cancer treatment to the population in greater Colombo area as well as to the patients referred by all other peripheral oncology units in the country. About 13,000 new cancer patients are registered at NCI annually. It has been recognized as a princi- 


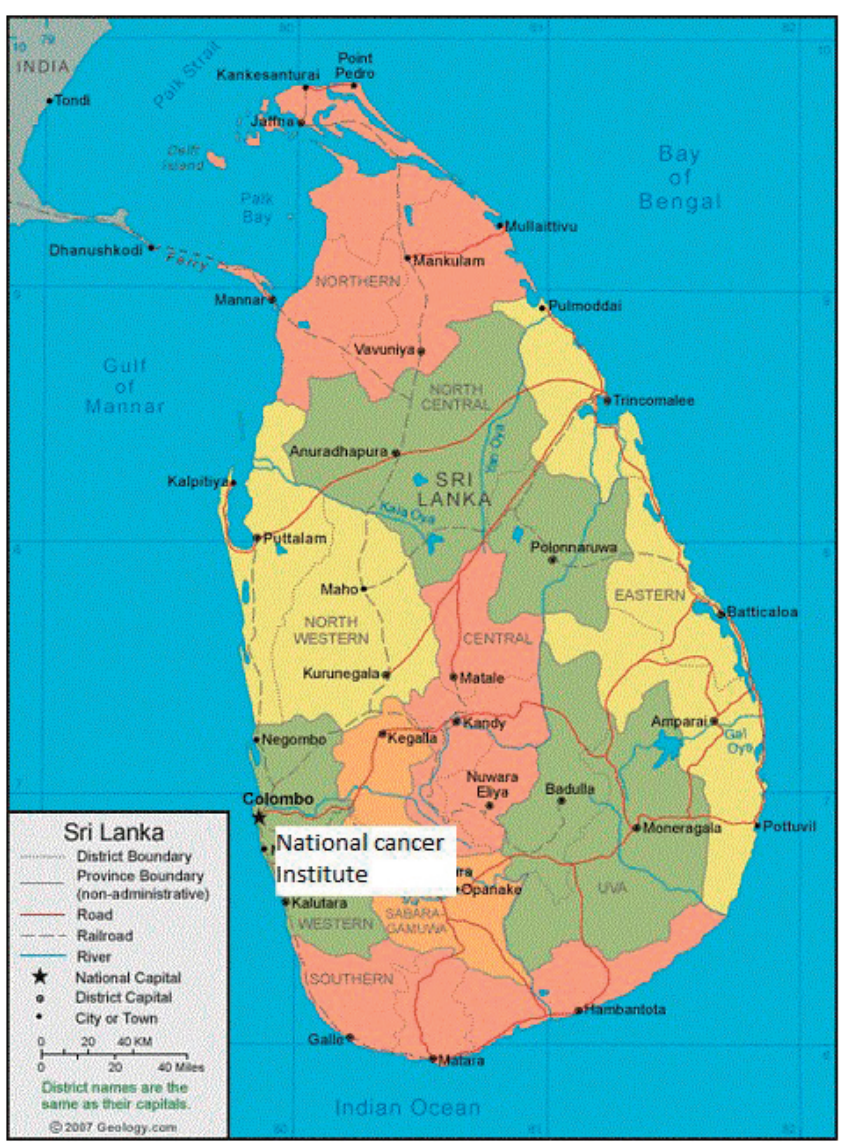

Figure 1. Sri Lanka

pal curative centre, postgraduate training unit and research institution in the field of oncology. About 1,100 new hematological malignancies are diagnosed in Sri Lanka each year, of which $25 \%$ are plasma cell disorders. Majority $(70 \%)$ of hematological malignancies diagnosed in Sri Lanka are treated at $\mathrm{NCI}^{2}$.

Absence of National Hematopoietic Stem Cell Transplant (HSCT) program in Sri Lanka was a major deficiency as most patients cannot afford transplant abroad. In 2013-14, clinicians at NCI decided to establish the first National HSCT center in collaboration with National Blood Transfusion Service (NBTS). This was enthusiastically supported by the government, local and expat sponsors. Collaboration between NCI and St Vincent's Hospital Sydney (SVHS), Australia followed by infrastructure development, mentorship, training and setup of protocols resulted in the first Autologous HSCT (AHSCT) in December 2016.

We describe the establishment of the first National HSCT center at NCI of Sri Lanka and the impressive results it achieved after the first 12 months.

\section{Methodology}

\section{Pre-transplant}

In 2014, NCI constructed a new Bone Marrow Trans-

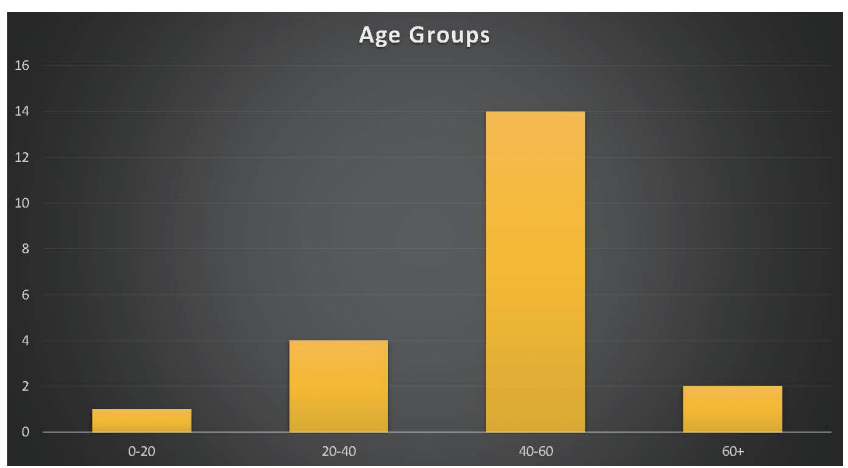

Figure 2. Age distribution

plant (BMT) unit which consisted of two rooms with provisions to expand to additional six rooms in future. Pathology, microbiology and infection control facilities were upgraded. NBTS undertook the responsibility of apheresis, stem cell processing and cryopreservation.

A team of 16 members consisting of clinicians, pathologists, nurses, scientists and pharmacists were trained at SVHS, Australia. This was followed by regular internetbased mentoring, establishing protocols, and on-site training by voluntary SVHS staff.

\section{Transplant eligibility}

Multiple myeloma (MM) patients in complete remission (CR), very good partial remission (VGPR) or partial remission (PR) and patients with relapsed NonHodgkin's lymphoma (NHL) and Hodgkin lymphoma (HL), who were less than 65 years with good performance status were eligible for the HSCT at the newly established unit of NCI.

\section{Stem cell mobilization}

Hematopoietic stem cells (HSC) were mobilized with cyclophosphamide and G-CSF in patients with MM. The mobilization regimen consisted of intravenous cyclophosphamide $\left(2 \mathrm{~g} / \mathrm{m}^{2}\right)$ on day 1 followed by subcutaneous filgrastim $10 \mu \mathrm{g} / \mathrm{kg}$ for eight consecutive days ${ }^{3}$. Apheretic stem cell collection was scheduled on next day. In HL and NHL patients, stem cells were collected after the last salvage chemotherapy regimen prior to transplant.

Peripheral blood CD34 + cell count was checked before the stem cell collection procedure and if the said count was below 15 cells/ $\mu \mathrm{L}$, the procedure was delayed. Second collection was carried out in cases where the first collection was suboptimal.

HSC products were cryopreserved, and post-thawed samples were checked for viability and contamination before product infusion.

\section{Conditioning and transplant}

Melphalan was used as the conditioning regime for 


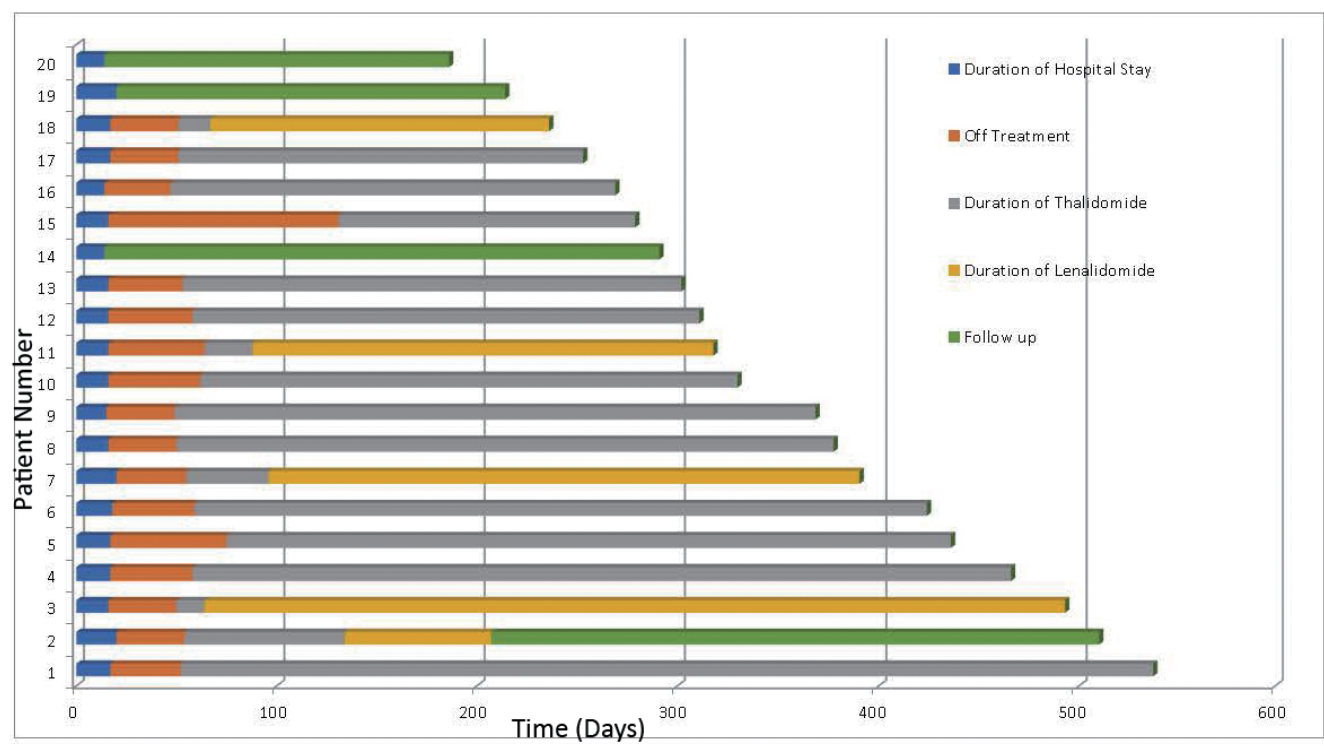

Figure 3. Summary of follow up data (Patient No.14-HL, Patient No. 19-POEMS, Patient No. 20-NHL, all others-MM)

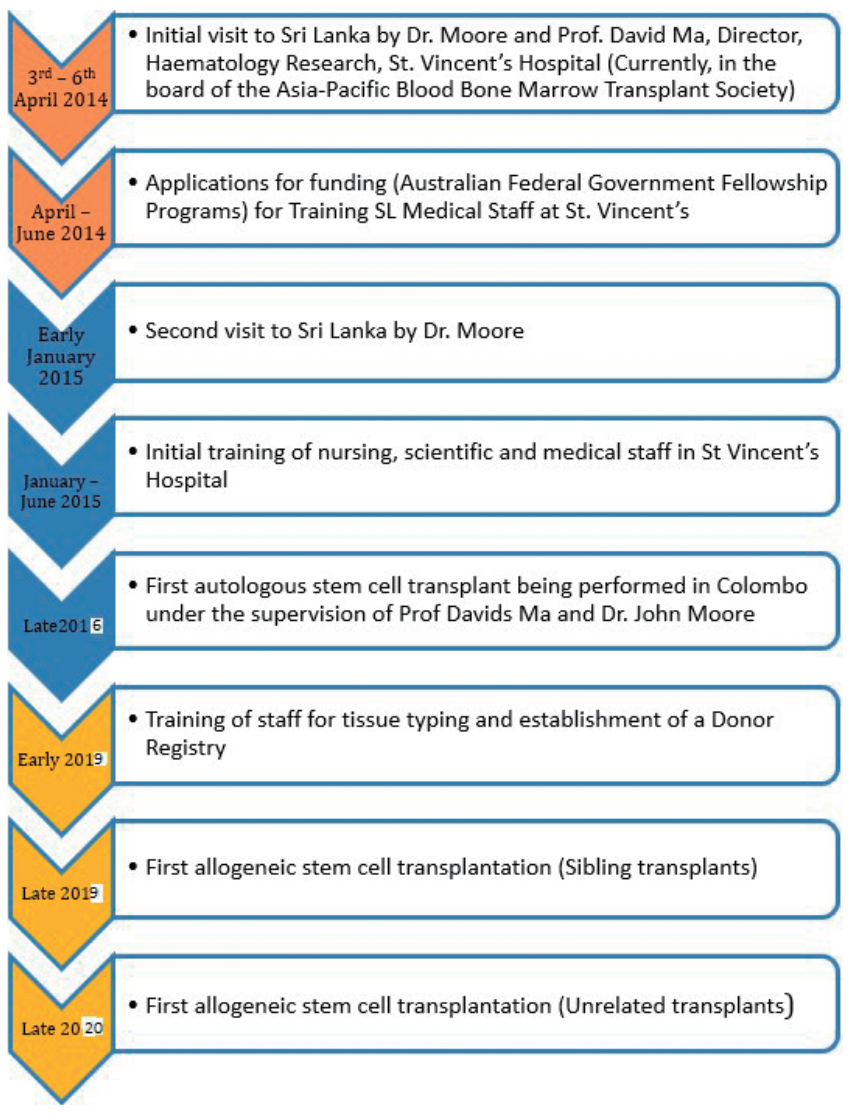

Figure 4. NCIM BMTU Time line

MM patients while BEAM regimen (carmustine, etoposide, cytarabine and melphalan) was used for the patients with $\mathrm{HL}$ and $\mathrm{NHL}^{4-8}$. Patients were kept under intensive monitoring peri-transplant. Immediate commencements of antibiotics, fluid management, blood component trans- fusions were instigated according to the protocols. Engrafted patients were discharged and managed regularly at the transplant clinic.

\section{Results}

Twenty autologous transplants were performed in the first year. Majority $(70 \%, \mathrm{n}=14)$ of patients were between 40 to 60 years (Figure 2). The mean age was 47 years (range: 17-62) and male to female ratio was $3: 2$. There were $17 \mathrm{MM}$ patients and one each of NHL (diffuse large B-cell lymphoma sub-type), HL and POEMS syndrome patients. All the patients were in ECOG performance status 0 at the time of transplant.

Eighty two percent $(n=14)$ of multiple myeloma patients have had induction triplet chemotherapy with bortezomib, thalidomide and dexamethasone (VTD), while the HL and the NHL patients had received ABVD and R-CHOP chemotherapy regimens, respectively. Both HL and NHL patients were given ESHAP (etoposide, methylprednisolone, cytarabine, cisplatin) chemotherapy regimen for their relapsed disease and both of them were in complete remission at the time of transplant. Sixty five percent $(n=11)$ of MM patients were in VGPR or better remission at the time of transplant.

The median value of CD34 + stem cells collected was $12.72 \times 10^{6} / \mathrm{kg}($ range: $3-31.7)$. Majority $(60 \%, \mathrm{n}=12)$ of patients needed a single apheresis and the maximum number of apheresis needed to collect the desired minimum CD34 + cell count was two. Median infused cell dose was $4.07 \times 10^{6} / \mathrm{kg}$ (range: $2.0-7.4$ ). The average difference between stem cell collection and stem cell transfusion was 33 days. 
The median engraftment day was 13 (range: 11-19) and the median hospitalization was 16 days (range: 14-20). All developed febrile neutropenia and seven patients showed positive blood cultures, out of which six showed growth of Gram-negative organisms. The most common pathogen isolated belonged to Pseudomonas species. The empirical broad-spectrum antibiotic combination used in febrile neutropenia was piperacillin-tazobactam and amikacin. All patients were routinely administered with intravenous immunoglobulin on day 6 posttransplant.

All patients developed Grade III thrombocytopenia, thereby needing platelet transfusions (median platelet packs transfused was 12). Zero transplant-related mortality (TRM) and acceptable morbidity was achieved.

Every multiple myeloma patient was prescribed maintenance treatment with thalidomide, and it was changed into lenalidomide when they developed unacceptable side effects. The median date of starting maintenance treatment was day 38 post-transplant. The most common side effects noted during the thalidomide maintenance were skin rashes, generalized itching and back pain.

At the median follow-up of 47 weeks (till 31/05/ 2018), the overall survival was $100 \%$ and all patients were still in remission (Figure 3).

\section{Conclusion}

After two and half years of initial planning of the HSCT centre at NCI, Sri Lanka, 20 transplants were performed during the first year of operation with an overall survival of $100 \%$.

This encouraging transplant outcome was attributed to commitment by trained multi-disciplinary team complemented by partnership with an experienced and dedicated voluntary mentoring team, meticulous planning, adequate equipment and infrastructure, support from the government, local and expatriate philanthropists (Figure 4).

We plan to initiate allogeneic stem cell transplant program at NCI and to assist the establishment of other HSCT centers in the country.

\section{Acknowledgements}

1. Support from The President of Sri Lanka, senior officials of Ministry of Health and Directors of NCI and NBTS

2. Staff of BMT unit, NCI

3. Helen Tao, Amanda McLaughlin, Annabel Horne and Staff of Department of Haematology and Bone Marrow Transplantation, SVHS, Australia

4. Jude Fernando, Chryshanthi Fernando and the mem- bers of the Australian Sri Lanka Society

5. Ahmad Tea, London and the local philanthropic supporters

\section{Conflict of Interest}

The authors declare no conflict of interest. Disclosure forms provided by the authors are available here.

\section{References}

1. Department of Census and Statistics-Sri Lanka, official web site. http://www.statistics.gov.lk/ [Accessed May 28, 2019]

2. Cancer Incidence Data Sri Lanka 2011. National Cancer Control Programme. Ministry of health, nutrition and indigenous medicine. $13^{\text {th }}$ publication. http://www.nccp.health.gov.lk/ images/PDF_PUBLICATIONS/Cancer_Incidence_Data2011. pdf [Accessed May 28, 2019]

3. Silvennoinen R, Anttila P, Säily M, Lundan T, Heiskanen J, Siitonen TM, et al. A randomized phase II study of stem cell mobilization with cyclophosphamide $+\mathrm{G}-\mathrm{CSF}$ or G-CSF alone after lenalidomide-based induction in multiple myeloma. Bone Marrow Transplant. 2015; 51: 372-376.

4. Moreau P, Facon T, Attal M, Hulin C, Michallet M, Maloisel F, et al. Comparison of $200 \mathrm{mg} / \mathrm{m}^{2}$ melphalan and 8 Gy total body irradiation plus $140 \mathrm{mg} / \mathrm{m}^{2}$ melphalan as conditioning regimens for peripheral blood stem cell transplantation in patients with newly diagnosed multiple myeloma: final analysis of the Intergroupe Francophone du Myelome 9502 randomized trial. Blood. 2002; 99: 731-735.

5. Palumbo A, Bringhen $\mathrm{S}$, and Bruno B. Melphalan $200 \mathrm{mg} / \mathrm{m}^{2}$ versus melphalan $100 \mathrm{mg} / \mathrm{m}^{2}$ in newly diagnosed myeloma patients: a prospective, multicenter phase 3 study. Blood. 2010; 115: $1873-1879$.

6. Mills W, Chopra R, McMillan A, Pearce R, Linch DC, Goldstone AH. BEAM chemotherapy and autologous bone marrow transplantation for patients with relapsed or refractory nonHodgkin's lymphoma. J Clin Oncol. 1995; 13: 588-595.

7. Caballero MD, Rubio V, Rifon J, Heras I, García-Sanz R, Vázquez L, et al. BEAM chemotherapy followed by autologous stem cell support in lymphoma patients: analysis of efficacy, toxicity and prognostic factors. Bone Marrow Transplant. 1997; 20: 451-458.

8. Jo JC, Kang BW, Jang G, Sym SJ, Lee SS, Koo JE, et al. BEAC or BEAM high-dose chemotherapy followed by autologous stem cell transplantation in non-Hodgkin's lymphoma patients: comparative analysis of efficacy and toxicity. Ann Hematol. 2008; 87: 43-48.

https://doi.org/10.31547/bct-2019-007

Copyright (C) 2019 APBMT. All Rights Reserved. 